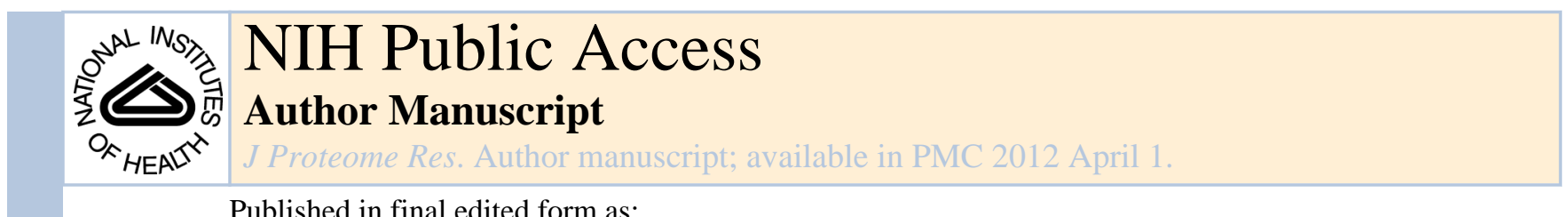

Published in final edited form as:

J Proteome Res. 2011 April 1; 10(4): 1621-1631. doi:10.1021/pr100974w.

\title{
Proteomic Discovery of Genistein Action in the Rat Mammary Gland
}

\author{
Jun Wang ${ }^{\star}, \dagger$, Angela M. Betancourt ${ }^{\dagger}$, James A. Mobley $\ddagger, \S$, and Coral A. Lamartiniere ${ }^{\dagger}, \S$ \\ Department of Pharmacology and Toxicology, the UAB Comprehensive Cancer Center, and \\ Division of Urology, Department of Surgery, University of Alabama at Birmingham, Birmingham, \\ Alabama 35294, United States
}

\section{Abstract}

Genistein, the primary isoflavone component of soy, consumed in diet during the prepubertal period suppresses chemically induced mammary cancer in rats. The current study used twodimensional gel electrophoresis (2-DE)/MS-based proteomic technology to identify proteins responsible for genistein breast cancer protection in vivo. Female offspring were exposed via lactating dams treated with $250 \mathrm{mg}$ genistein/kg AIN-76A diet from days 1 to 21 postpartum (prepubertal period). Mammary glands were collected at 21 and 50 day of age and subjected to 2DE/MS and immuno-blot analyses. Twenty-three proteins were determined to be differentially regulated $(\mathrm{p}<0.05)$ and identified using 2-DE, followed by MALDI-TOF/TOF or LC-ESI-MS/MS. Five of these proteins were validated by immuno-blots. Annexin A2 was significantly increased at 21 days, yet found to be decreased at 50 days. Fetuin B was found to be unchanged at day 21, but increased at day 50. Phosphoglycerate kinase 1 (PGK1) was unchanged at day 21, but decreased at day 50. Gelsolin was increased at day 21, but not at day 50. Protein disulfide-isomerase A3 (PDIA3) was decreased at day 21 and unchanged at day 50. Also, we found that vascular endothelial growth factor receptor 2 (VEGF-R2) and epidermal growth factor receptor (EGF-R) were decreased in mammary glands of 50-day-old rats treated prepubertally with genistein. This study demonstrates the usefulness of proteomics for the discovery of key proteins involved in signaling pathways to understand genistein mechanisms of action in breast cancer prevention.

\section{Keywords}

Genistein; Mammary Glands; Chemoprevention; 2-DE/MS; Proteomics

\section{Introduction}

Epidemiological evidence, together with preclinical data from in vitro and animal studies, strongly supports a correlation between soy isoflavone consumption and protection against breast cancer. The biological processes modulated by isoflavones, and especially by genistein, have been extensively studied, yet without leading to a clear understanding of the cellular and molecular mechanisms of action involved. Many cancer causation and prevention studies involving environmental chemicals have investigated direct effects in adult animals, because cancer is considered a disease associated with aging. However,

\footnotetext{
*Corresponding Author: Dr. Jun Wang, Department of Pharmacology and Toxicology, University of Alabama at Birmingham, 1670 University Boulevard, Birmingham, AL 35294, USA. Tel 205-934-7137. Fax 205-934-8240. jun.wang@ccc.uab.edu.

Department of Pharmacology and Toxicology, University of Alabama at Birmingham.

\$Division of Urology, Department of Surgery, University of Alabama at Birmingham.

$\S_{\mathrm{UAB}}$ Comprehensive Cancer Center, University of Alabama at Birmingham.
} 
exposure to environmental chemicals during critical periods of early development plays an important role in breast cancer susceptibility in adulthood. ${ }^{1}$

Our laboratory has focused research on the chemopreventive nature of the soy isoflavone genistein. We demonstrated that prepubertal exposure to genistein decreases tumor multiplicity and diminishes incidence of adenocarcinomas in the dimethylbenz[a]anthracene (DMBA) model of mammary cancer. ${ }^{1,2}$ Whole-mount analysis of mammary glands showed that prepubertal exposure to genistein enhances mammary gland differentiation, that is, results in fewer terminal end buds and more lobules in adult rats. ${ }^{2}$ Terminal end buds are the least mature structures and are the most susceptible to chemical carcinogens. ${ }^{3}$ The resulting morphological action of genistein can be characterized as enhancing gland maturation. This maturation yields a gland that is ultimately less proliferative and less susceptible to chemical carcinogenesis. ${ }^{2-5}$ In general, the morphological development of the mammary gland extends beyond the embryonic stage and largely occurs during postnatal development. The postnatal development of the rat mammary gland closely resembles that of the human and provides a meaningful model for studying cancer chemoprevention. ${ }^{6}$

We have hypothesized that genistein exerts its chemopreventive actions by postnatally programming developmental modifications to genes/proteins that render the mammary gland less susceptible to cancer. Proteomic techniques provide a high throughput platform for comparative and functional proteome research. To elucidate the changes in protein expression modulated by exposure to genistein in vivo, we adopted the technologies of 2dimensional gel electrophoresis (2-DE)/MS-based proteomic approach to find new biomarkers of genistein action in the rat mammary gland. In the present study, we discovered, via 2-DE/MS and immuno-blot analyses, differential protein expressions of annexin A2, fetuin B, gelsolin, PDIA3 and PGK1 in the mammary glands of rats exposed to genistein. We also constructed a possible mechanism of chemoprevention by changes in the possible targets of its action, vascular endothelial growth factor receptor 2 (VEGF-R2), EGF-R and p-Akt, and showed how these changes in protein expression may be involved in the chemopreventive action of genistein.

\section{Materials and Methods}

\section{Animals}

Animal care and treatments were performed according to established guidelines, and protocols were approved by the UAB Animal Care Committee. Seven week old female Sprague-Dawley rats were purchased from Charles River Laboratories (Wilmington, MA). They were housed in a temperature controlled facility with a 12-h light/dark cycle, bred with proven Sprague-Dawley studs in our facilities and fed phytoestrogen-free AIN-76A diet (Harlan Teklad, Madison WS) during mating. On the day of birth (designated as postnatal day zero), offspring were sexed and litters were culled to 10 offspring per lactating dam. The lactating dams were treated with either $250 \mathrm{mg}$ genistein (Roche, Basel, Switzerland) $/ \mathrm{kg}$ AIN-76A diet or AIN-76A diet only as controls from birth until 21 days of age (prepubertal genistein exposure). Offspring were weaned at day 21 and continued on AIN-76A diet only. Female rats were killed on day 21 or $50 \pm 1$ (prepubertal and young adult rats, respectively). At day $50 \pm 1$, female offspring were killed in the estrous phase. At the time of death, the fourth abdominal mammary glands were rapidly dissected from live ketamine/xylazine anesthetized animals (to minimize proteolysis) and snap-frozen at $-80{ }^{\circ} \mathrm{C}$ or put in $10 \%$ formalin for later analyses. Three unique sets of genistein-and control-treated animals were generated for these experiments. Set 1 was used for the production of 2-DE (8-10 rats/ group); set 2 was used for validation studies via western blot analysis (8 rats/group); set 3 was used for immunohistochemistry (6 rats/group). 


\section{Sample Preparation for 2-DE}

Frozen mammary glands were ground in liquid nitrogen and homogenized in deionized lysis buffer containing $2 \mathrm{M}$ thiourea, $7 \mathrm{M}$ urea, $4 \%$ w/v CHAPS, Pharmalyte 3-10 and protease inhibitor mix (Amersham Biosciences, Piscataway, NJ) followed by homogenization. Sample homogenates were incubated on ice for one hour and centrifuged at $16,000 \times \mathrm{g}$ for 20 minutes at $4^{\circ} \mathrm{C}$. Protein concentrations of the supernatants were determined with the Bradford assay (BioRad). Equal concentration of protein per sample ( $800 \mu \mathrm{g})$ was loaded onto the albumin and $\operatorname{IgG}$ removal kit following the manufacturer protocol (GE Healthcare). The albumin and IgG depleted samples were concentrated and purified by overnight acetone precipitation. Protein pellets were re-suspended in lysis buffer and centrifuged at $6,000 \times \mathrm{g}$ for 20 minutes. Protein concentrations of the supernatants were determined with the Bradford assay (BioRad). Eighteen cm IPG strips (GE Healthcare), over the $\mathrm{pH}$ range of 4-7, were passively rehydrated overnight at room temperature with $340 \mu$ of rehydration solution containing $150 \mu \mathrm{g}$ protein and rehydration buffer $(2 \mathrm{M}$ thiourea, $7 \mathrm{M}$ urea, $4 \% \mathrm{w} / \mathrm{v}$ CHAPS, $0.3 \% \mathrm{w} / \mathrm{v}$ DTT, $0.5 \%$ v/v IPG buffer $\mathrm{pH} 3-10$ ). Samples were randomized for placement in the rehydration tray. The IPG strips were placed on a flatbed electrophoresis unit (Multiphor II) and electro-focused as follows: (1) $500 \mathrm{~V}$ for $1 \mathrm{~h}$, (2) $3500 \mathrm{~V}$ for $1.5 \mathrm{~h}$, and (3) $3500 \mathrm{~V}$ for $22.5 \mathrm{~h}$. Prior to second dimension separation the focused strips were equilibrated in the equilibration buffer $(50 \mathrm{mM}$ Tris- $\mathrm{HCl} \mathrm{pH} \mathrm{8.8,} 6 \mathrm{M}$ urea, $20 \% \mathrm{v} / \mathrm{v}$ glycerol, $2 \% \mathrm{w} / \mathrm{v}$ SDS, $20 \mathrm{mM}$ DDT, $0.01 \% \mathrm{w} / \mathrm{v}$ bromophenol blue) for $20 \mathrm{~min}$ followed by a second equilibration in the equilibration buffer containing $4 \% \mathrm{w} / \mathrm{v}$ iodacetimide instead of DTT for 20 min. Equilibrated IPG strips were overlaid onto a precast $1.5 \mathrm{~mm}, 12.5 \%$ SDS gels (Bio-Rad, Hercules, CA) and run on a Protean Plus ${ }^{\mathrm{TM}}$ Dodeca $^{\mathrm{TM}}$ cell vertical electrophoresis unit according to manufacturer's suggestions. Both IEF and SDS gels were run as block groups consisting of equal treatments per run. Once gels were run to completion, they were fixed, stained using SYPRO® Ruby protein gel stain (Biorad), and de-stained before being imaged.

\section{2-DE Image Acquisition and Analysis}

Images of gels stained with SYPRO® Ruby were acquired on a Typhoon Trio+ Variable Mode Imaging System (GE Healthcare), using the specific laser band-pass filter for SYPRO ${ }^{\circledR}$ Ruby excitation and emission wavelength $(610 \mathrm{~nm})$. Gels were scanned individually and Photo Multiplier Tube (PMT) voltage was adjusted for maximum image quality with minimal signal saturation. Images were checked for saturation during the acquisition process using ImageQuant ${ }^{\mathrm{TM}} \mathrm{TL}$ software (GE Healthcare). Images were stored as 16-bit GEL files, and exported into Progenesis SameSpots software v.3.0, 2008 (Nonlinear Dynamics, Newcastle, UK) image analysis software. Eight to ten biological replicate gel images from each treatment group were aligned and analyzed using Progenesis SameSpots workflow. The gel with the spots pattern that was similar to the majority of the other gels was chosen as reference gel. Every gel was independently matched to this reference gel. Gel images were aligned by automated calculation of alignment vectors after manually assigning 10 landmark vectors. Artifacts or spots that could not be verified as true matches were disregarded, and misalignments were corrected by manual warping when appropriate. The aligned images were grouped into their corresponding treatment group and the statistically ranked list of spots was evaluated in the review stage of the Progenesis SameSpots software based on ANOVA p-value.

\section{In-gel Trypsin Digestion}

SYPRO® Ruby stained spots that showed differential expression were excised precisely with a scalpel, diced into $1 \mathrm{~mm}$ pieces, and placed in labeled Eppendorf tubes. In addition, blank gel pieces from a spot-free region, and reference spots (known marker proteins from the 2-DE) were excised and served as controls for trypsin digestion and were run in parallel 
with the protein spots of interest. The gel pieces were allowed to equilibrate with $100 \mu$ of $100 \mathrm{mM}$ ammonium bicarbonate for $15 \mathrm{~min}$. After this incubation, the liquid was removed and replaced with $100 \mu 150 \% \mathrm{ACN}$ (accenture), $50 \mathrm{mM}$ ammonium bicarbonate for $15 \mathrm{~min}$, followed by incubation with $50-100 \mu 1100 \%$ ACN for 10 min or until all gel slices turned white. After removal of ACN, gel slices were desiccated in a vacuum centrifuge for $5 \mathrm{~min}$, and digested overnight at $37^{\circ} \mathrm{C}$ with sequencing-grade trypsin $(10 \mu \mathrm{g} / \mathrm{ml}$ trypsin dissolved in $25 \mathrm{mM}$ ammonium bicarbonate). The next day, the supernatants were then transferred to new labeled Eppendorf tubes, and the remaining peptides were further extracted $2 \mathrm{x}$ by adding $15-25 \mu \mathrm{l}$ of $60 \% \mathrm{ACN}, 0.1 \%$ TFA (trifluoroacetic acid) to each tube containing the gel slices, and extracted peptides were combined with the supernatant collected after trypsin digestion. The combined supernatant-extract mix was concentrated to near dryness in a vacuum centrifuge. Extracted peptides were reconstituted in $20 \mu 10.1 \%$ TFA, 50\% ACN, or $10 \mu 10.1 \%$ TFA for MALDI and LC/MS analysis, respectively.

\section{MALDI Mass Spectrometry Analysis}

MALDI-TOF/TOF analysis was performed using a Bruker Ultraflex III MALDI-TOF/TOF (Bruker Daltonics, Bremen, Germany) operated in the reflector mode for MALDI-TOF peptide mass fingerprint (PMF) combined with LIFT mode for peptide characterization by tandem MS(MS). FlexControl was utilized to control the instrument while linked dynamically to FlexAnalysis and BioTools software packages (Bruker Daltronics), which utilize MASOT (Matrix Sciences, London, UK) to identify peptide fragment patterns through the SwissProt MSDB 20051115 rodent specific database. Extracted peptides $(2 \mu \mathrm{l})$ were directly applied onto the target (AnchorChip ${ }^{\mathrm{TM}}$, Bruker Daltonics, Bremen, Germany) that was mixed with $2 \mu \mathrm{l}$ of the matrix (CHCA [ $\alpha$-Cyano-4-hydroxycinnamic acid], $10 \mathrm{mg}$ / $\mathrm{ml}$ in $0.1 \% \mathrm{TFA} / 50 \% \mathrm{ACN}$, and $5 \mathrm{mM}$ ammonium citrate), and air dried. More specifically, samples were analyzed by one summed PMF spectra consisting of 1000 shots, followed by up to 20 additional MS/MS analysis consisting of 2000 summed shots acquired for the top intensity peptides when present. Calibration for PMF samples (digests) was performed externally using a mixture of nine peptides ranging from $\mathrm{m} / \mathrm{z} 757.40$ to 3147.47 , spotted directly adjacent to each sample analyzed. The $m / z$ range was 700-4000 for PMF and 402560 for MS/MS, and spectra were interpreted with the FlexAnalysis by applying a signalto-noise $(\mathrm{s} / \mathrm{n})$ ratio threshold set at three. The set parameters for database searching included trypsin with one maximum missing cleavage site, and a mass tolerance of $25 \mathrm{ppm}$ for PMF, with an MS/MS tolerance of $0.5 \mathrm{Da}$, a fix modification of carbamidomethyl(C), and a variable modification of oxidation of methionine and variable phosphorylation at (Tyr, Thr, and Ser) were considered.

\section{LC-ESI-MS/MS Analysis}

Searches for protein identifications were carried out using species-specific subsets of the UniRef database. All tandem mass spectrometry data was converted to mzXML format using instrument-specific converting software packages (http://proteinprophet.sourceforge.net/prot-software.html; Institute for Systems Biology "ISB", Seattle Washington \& Fred Hutchinson Cancer Center) and run through SEQUEST, $\mathrm{X}$ !TANDEM, and MASCOT separately. X!TANDEM was downloaded from The Global Proteome Machine Organization (http://www.thegpm.org/TANDEM), while licenses were purchased for the other two search engines (Thermo for SEQUEST, and Matrix Science for MASCOT). All three of these top matching algorithms were utilized in order to increase confidence in protein identifications, while also decreasing the propensity for false negatives. An example of specifics for each matching program include 1) SEQUEST which is the only algorithm that takes into account relative, and absolute intensity values generated from each peptide, 2) X!TANDEM, which takes into account partial digests with a focus on $\mathrm{B}$ and $\mathrm{Y}$ ions, and 3) MASCOT, which takes into account the database size when calculating 
a unique match. The databases utilized are the same as referenced in the MALDI-MS analysis. This data is then "combined" and analyzed using peptide and protein Prophet (also from ISB, above), which is capable of utilizing all of this data from each output to determine a "best fit" for a specific peptide fragmentation pattern as it relates to an appropriate match from a large database with high confidence. Cut off filters for both peptide and protein Prophet vary depending on a dynamically generated probability score that is determined based on each data set. In addition, this approach calculates a true positive correlation as opposed to simply a false positive, common to other approaches. This is important since sample characteristics can change from run to run and since Prophet takes into account true and false-positives, a more accurate probability score can be determined.

\section{Annotations and Gene Ontology Mapping}

The LTQ and MALDI generated output files were analyzed and filtered as described above. This resulted in a list of UniRef referenced ID's, which were re-annotated (mapped) first to an Entrez Gene file format, followed by the generation of a Gene Ontology Enrichment output file, which maps all proteins to previously referenced cellular components. This was carried out using PANTHER (Protein ANalysis THrough Evolutionary Relationships) system (http://www.pantherdb.org). Subcellular localization (GO cellular component) was annotated manually based on literature searches (UniProt, ExPASy, and NCBI Entrez). Protein classification led to the summation of total proteins known to be associated with each biological process, pathway, and sub-cellular localization. The summed data was graphed as pie charts using Origin 7.0 (OriginLab Corporation, Northampton, MA).

\section{Immunoblotting}

To determine the changes in protein expression, 8 mammary gland samples per treatment group were analyzed by western blots. The same quantity of protein from each sample was separated by SDS-PAGE and transferred to a nitrocellulose membrane (Bio-Rad, Hercules, CA). The membranes were blocked and immunoblotted with appropriate antibodies including anti-annexin A2 (R\&D Systems, Minneapolis, MN), anti-fetuin B (Santa Cruz, CA), anti-gelsolin (abcam, Cambridge, MA), anti-PDIA3 (Cell Signaling, Danvers, MA), anti-PGK1(R\&D Systems, Minneapolis, MN), anti-VEGF-R2 (abcam, Cambridge, MA), anti-p-Akt (Cell Signaling, Danvers, MA) and anti-EGF-R (Cell Signaling, Danvers, MA). Molecular weight ladders (Bio-Rad) and reference proteins from the respective companies were used as positive controls. Nitrocellulose membranes were incubated with an appropriate secondary antibody conjugated to HRP (Cell Signaling Technology), followed with a chemiluminescent substrate (Pierce) and exposed to X-ray radiography film. Quantitative analysis of protein expression was accomplished by scanning autoradiogram and densitometry (ImageJ, NIH).

\section{Immunohistochemistry}

Immunohistochemistry (IHC) was employed to measure Ki-67 antigen as a marker for proliferating cells in mammary glands of 21 and 50 day old rats ( 6 controls, 6 genisteintreated). After blocked in $2.5 \%$ normal horse serum, specimens were incubated with monoclonal mouse anti-rat Ki-67 antigen, clone MIB-5 antibody (Dako Cytomation, Carpinteria, CA) diluted in PBS (phosphate buffered saline) with 1\% BSA (bovine serum albumin) for $30 \mathrm{~min}$. Chromogen, diaminobenzidine (DAB) (Vector) was applied to samples for 10 min followed by a wash in tap $\mathrm{H}_{2} \mathrm{O}$ for 5 min. To counter-stain, hematoxylin QS (Vector) was applied to the specimens for one minute followed by a wash in tap $\mathrm{H}_{2} \mathrm{O}$. The slides were viewed using a Nikon Labophot-2 microscope (Nikon Corporation, Tokyo, Japan) and data digitally recorded using a Nikon 8.0 Mega Pixels CoolPix 8700 Digital Camera (Nikon). The pictures were counted using Image J software (Image J, NIH). The epithelial cells positive for Ki67 (stained brown) were counted as well as the non- 
proliferative epithelial cells (stained blue). A proliferative index was given by the number of positively stained cells divided by the total number of cells counted X 100 .

\section{Statistical Analysis}

Statistical analyses of immunoblotting and immunohistochemistry were performed by Fisher's exact test to determine significance $(\mathrm{P}<0.05)$. Analyses were conducted using Microsoft Office Excel 2003 (Microsoft Corp., Seattle, WA). For the biochemical data, experiments were analyzed using one way analysis of variance (ANOVA), with subsequent multiple comparisons. The $\mathrm{p}$ values associated with the individual comparisons were completed using separate t-tests.

\section{Results}

\section{Effects of Genistein on the Proteome of Rat Mammary Glands}

Proteins from mammary glands of 21 and 50 day old rats were evaluated by 2-D gel electrophoresis. Using comparative proteomics with Progenesis SameSpots software, we analyzed the protein profiles between genistein exposed and unexposed mammary glands. Figure 1 shows representative 2-D gel of protein profiles after Sypro Ruby staining. Statistical analysis of the normalized volume of matched spots performed using SameSpots software revealed 10 and 16 protein spots that were significantly changed in mammary glands of 21 and 50 day old rats, respectively $(\mathrm{p}<0.05)$ (Tables 1 and 2). Three of these protein spots (peroxiredoxin-2, PDIA3 and Vitamin D-binding protein) were found to be differentially abundant in the mammary glands of both 21 and 50 day old rats.

\section{Identification of Genistein-regulated Proteins by MALDI-TOF/TOF or LC-MS/MS}

Differentially regulated protein spots from mammary glands of genistein-treated rats compared to controls were excised from the SYPRO-stained gels and subjected to MALDITOF/TOF or LC-ESI-MS/MS analyses. Tables 1 and 2 provide the Swiss-Prot accession numbers, MASCOT scores (for proteins identified by MALDI-TOF-TOF), xCorr score (for proteins identified by LC-MS/MS), percentage of sequence coverage, fold change, ANOVA, postnatal age, theoretical molecular weight $(\mathrm{kDa}), \mathrm{pI}$ and brief description of molecular function. Following protein identification, they were categorized according to 1) biological processes, 2) signaling pathway, and 3) cellular localization using the PANTHER gene ontology database (http://pantherdb.org) (Figure 2).

\section{Validation of Differentially Regulated Proteins by Immuno-blot Analysis}

Based on normalized spot volume, the expressions of 26 protein spots were found to differ significantly between the genistein-treated and the control animals $(P<0.05)$ (Tables 1 and 2 ). For those proteins that we were able to obtain commercially available antibodies, we pursued protein validation by immuno-blot analysis. Changes were validated for annexin A2, fetuin B, gelsolin, PDIA3 and PGK. Consistent with the 2-DE/MS data, immuno-blot analysis showed that annexin A2 and PGK were significantly decreased in mammary glands of 50 day old rats following prepubertal exposure to genistein with decreases of $66 \%$ and $46 \%$, respectively (Figures $3 \mathrm{~A}$ and C). The protein fetuin B was validated and increased $(67 \%)$ in mammary glands of 50 day old rats by western blots. Also, we validated the increase of gelsolin (96\%) and decrease of PDIA3 (32\%) in 21 day old rats (Figures 3A and B). Since annexin A2 was found to be differentially decreased in 50 day old rats exposed to genistein by 2-DE/MS and immuno-blot analyses, we also measured annexin A2 expression in mammary glands of 21 day old rats. We found that prepubertal genistein exposure resulted in a significant increase (230\%) in annexin A2 in mammary glands of 21 day old rats (Figures $3 \mathrm{~A}$ and $\mathrm{B}$ ). 
In order to gain insight of up-stream and down-stream signaling from annexin A2 we measured low abundance proteins whose regulation and mechanisms could be associated with cell proliferation and chemoprevention. In mammary glands of 50 day old rats, but not in 21 day old rats, VEGF-R2 and EGF-R were significantly decreased (Figure 4). In contrast, in mammary glands of 21 day old rats, but not in 50 day old rats, p-Akt was increased.

\section{Effects of Genistein on Cell proliferation in Rat Mammary Glands}

$\mathrm{Ki}-67$ antigen staining was used as an indicator of proliferating cells in rat mammary glands. Prepubertal administration of genistein resulted in a statistically significant reduction of the proliferation rate from $27.3 \%$ to $11.6 \%$ in mammary glands of 50 day old rats $(P=0.0006)$. At 21 days, no statistically significant difference among the groups was seen (Figure 5).

\section{Discussion}

Our laboratory previously reported that pubertal exposure of female rats to genistein via the diet, prior to puberty, resulted in protection from dimethylbenz $[a]$ anthracene-induced mammary cancer. ${ }^{2}$ The protection was ascribed to early postnatal genistein exposure enhancing mammary gland maturation, i.e. enhancing the maturation of terminal end buds to lobules. The former are the most susceptible terminal ductal structures of the mammary glands while the latter are more mature and resistant terminal ductal structures to chemical carcinogenesis due to their high degree of differentiation. ${ }^{3,7}$ The chemopreventive effect of accelerated glandular differentiation by genistein is similar to the protection derived from pregnancy early in life in humans, ${ }^{8}$ whereby hormonal exposure results in mammary gland maturation.

The biological processes modulated by genistein have been extensively studied, yet without leading to a clear understanding of the cellular and molecular mechanisms of action. To enhance our ability to discover biochemical pathways that genistein may affect in vivo, we adopted the technologies of 2-DE/MS to find new biomarkers of genistein action in the rat mammary gland. In our study, we discovered 23 unique proteins whose expressions were significantly altered by prepubertal genistein treatment in mammary glands of 21 and 50 day old rats. An ontology analysis of these proteins revealed that they were mainly involved in multiple key cellular processes, such as metabolism, cell structure, cell motility, cell cycle and transport. The sorting of these differentially expressed proteins into several functional groups permits the development of hypotheses concerning their potential involvement, at least in part, in underlying the molecular mechanisms of cell growth inhibition in mammary glands of rats treated with genistein.

Based on commercial availability, 10 specific antibodies were employed to validate the proteins which were found to be significantly altered by 2-DE and MS identification. By using new mammary samples of identically treated rats, we were able to validate annexin A2, fetuin B, gelsolin, PDIA3 and PGK1 by immuno-blot analysis.

Annexin A2, a cytosolic phospholipid and $\mathrm{Ca} 2+$ binding protein, is a receptor of many angiogenesis-related proteins, such as angiostatin and tissue plasminogen activator (t-PA). Annexin A2 can form heterotetrameric complexes on the surface of endothelial cells with the annexin A2 light chain (called S100A10 or p11), and this stimulates generation of t-PA dependent plasmin..$^{9}$ Plasmin is a highly reactive enzyme that is physiologically involved in fibrinolysis and plays an important role in neoangiogenesis. ${ }^{10}$ In addition, annexin A2 is a substrate of PKC $\alpha, \mathrm{PKC} \beta \mathrm{I}$, and PKC $\beta \mathrm{II}$ kinases in cells. Phosphorylation of annexin A2 serine 25 is associated with its nuclear entry, DNA synthesis, and cell proliferation ${ }^{11}$. Increased expression of annexin A2 has been described in several types of tumors, including 
breast cancer, ${ }^{12}$ and down-regulation of annexin $\mathrm{A} 2$ has been reported to decrease cell proliferation in MCF-7/ADR cells. ${ }^{13}$ We found annexin A2 expression to be increased in the mammary glands of 21-day-old but decreased in 50 day old rats with only prepubertal exposure to genistein. Increased annexin A2 at day 21 may be due to direct action of genistein, while at day 50 a decrease in annexin A2 may be interpreted as a developmental response to the prepubertal exposure. Up-regulated annexin A2 and phospho-Akt at day 21 also suggests that altered cell proliferation and survival during the prepubertal period could enhance normal mammary gland maturation and differentiation and eventually lead to altered response in later life of developing offspring. This is evident at day 50 where the expression of annexin A2 was decreased as compared to being increased at day 21.

Given a potential link with annexin A2 and VEGF-R2 in angiogenesis, ${ }^{9}$ and annexin A2 and EGF-R in cell proliferation, ${ }^{14,15}$ we conducted analyses for protein expression of VEGFR2 and EGF-R. The results showed a significant decrease in VEGF-R2 and EGF-R expression in the mammary glands of 50-day-old rats with only prepubertal genistein treatment. These results complemented the finding that Annexin A2 level was reduced in the mammary glands of 50-day-old rats following prepubertal genistein treatment. Observed decreases in Annexin A2, VEGF-R2 and EGF-R at time of carcinogenesis could reduce cell proliferation, angiogenesis and cell invasion, and favor breast cancer prevention.

The cystatin-like gene family (Kng, Ahsg/fetuin, Fetuin-B, and Hrg) is implicated in blood clotting, tumorigenesis, and angiogenesis. Fetuin-B is unambiguously a paralogue of Ahsg/ fetuin. ${ }^{16}$ The name fetuin has been suggested as the universal nomenclature for this protein in every species since they originate from a single ancestral gene and are counterparts of a single protein, i.e. orthologues in different species. ${ }^{17}$ Several studies have implicated the various members of this cluster of cystatin-like family genes in tumor development, particularly in angiogenesis. Kng and Ahsg/fetuin can exhibit anti-angiogenic properties by inhibiting proliferation and inducing apoptosis of human endothelial cells. ${ }^{18,19} \mathrm{Ahsg} / \mathrm{fetuin}$ is known to bind and inhibit the tyrosine kinase activity of the insulin receptor. ${ }^{20}$ It has been demonstrated that over-expression of fetuin-B in skin squamous carcinoma cells leads to suppression of tumor growth in nude mice, suggesting a tumor suppressor activity. ${ }^{21}$ The observed increase in fetuin-B at day 50 indicates a potential role in suppressing breast cancer.

PGK1 functions as an adenosine triphosphate (ATP)-generating enzyme of the glycolytic pathway which catalyzes the conversion of 1,3-diphosphoglycerate to 3-phosphoglycerate and as a secreted protein by tumor cells to participate in the angiogenesis process as a disulfide reductase. ${ }^{22}$ PGK1 also influences DNA replication and repair in mammalian cell nuclei ${ }^{23}$ and induces multidrug resistance (MDR) through an MDR-1-independent mechanism. ${ }^{24}$ PGK1 is involved in the onset of malignancies, including breast cancer. ${ }^{25} \mathrm{We}$ found reduced PGK1 in mammary glands at day 50, supporting the potential of genistein to suppress breast cancer.

Gelsolin has been the subject of more intensive study in terms of its role in tumorigenesis. ${ }^{26}$ While several reports indicate that gelsolin acts either as a tumor suppressor $27,28,29$ or as a tumor activator, ${ }^{30,31}$ it may play a dual role in carcinogenesis. Protein disulfide isomerase family A, member 3 (PDIA3) also known as glucose-regulated protein, 58-kD(GRP58) is an isomerase enzyme. Expression analysis using matched human tumor/normal array revealed an up-regulation of GRP58 in breast, uterus, lung, and stomach tumors compared with normal tissues of similar origin. ${ }^{32}$ We found increased gelsolin and reduced PDIA3 in mammary glands of 21 day old rats, but unchanged at day 50. The significance of genistein action on gelsolin and PDIA3 in the mammary glands of rat needs to be further investigated. 
While we do not know if genistein alters the amount or composition of the milk, we have previously reported that the total genistein concentration was 11-12-fold higher in the stomach milk of neonates compared to the mother's milk. ${ }^{2}$ In the dam's milk, $43 \%$ of the genistein was conjugated, while in the 7-day-old offspring's stomach milk only 3-22\% of the genistein was conjugated. Furthermore, it has been reported that dietary genistein at 25, 250 , and $1250 \mathrm{mg} / \mathrm{kg}$ AIN-76A diet fed to pregnant rats, beginning on gestational day 7 and the offspring continued until postnatal day 77 , did not significantly alter gestational duration, total offspring/litter, total sex ratio, live pups/litter, live sex ratio, or average weight/live pup. ${ }^{33}$ There was no significant effect on open field activity, play behavior, day or night running wheel activity, or intake of water and saccharin-flavored solution. Flynn has also reported that lifelong and multigenerational exposure to dietary genistein had no severe effects on nursing behavior in rats. 33

In summary, data from this and previous work suggest that early postnatal (prepubertal) exposure to genistein enhances mammary gland maturation possibly by up-regulating annexin A2, the Akt-signaling pathway and GTP-cyclohydrolase 1 (GTP-CH1) ${ }^{1,2,34}$ during the prepubertal period and hence, cell differentiation later in life. This unique developmental maturation leads to a new biochemical blueprint, whereby the cells now have reduced annexin A2, EGF-signaling, VEGF-R2, PGK1 and increased fetuin B in adults, which renders the mature mammary gland less proliferative and less susceptible to chemically induced mammary cancer initiation, angiogenesis, and cancer progression. Therefore, genistein acts through a diverse and coordinated effect of signaling mechanisms and pathways that likely account for the cellular changes responsible for its chemopreventive action. Knowledge gained from these experiments provides more information on the possible mechanisms of action of genistein that result in chemoprevention. Future work will examine other members of these signaling pathways more closely and address the potential mechanisms involved.

\section{Acknowledgments}

This study was supported by the Breast Cancer and the Environment Research Centers grant number U01 ES/CA ES012771 from the National Institute of Environmental Health Sciences (NIEHS), and the National Cancer Institute (NCI), NIH, DHHS.

\section{References}

1. Lamartiniere CA. Timing of exposure and mammary cancer risk. J Mammary Gland Biol Neoplasia. 2002; 7:67-76. [PubMed: 12160087]

2. Fritz WA, Coward L, Wang J, Lamartiniere CA. Dietary genistein: perinatal mammary cancer prevention, bioavailability and toxicity testing in the rat. Carcinogenesis. 1998; 19:2151-8. [PubMed: 9886571]

3. Russo J, Wilgus G, Russo IH. Susceptibility of the mammary gland to carcinogenesis. Am J Pathol. 1979; 96:721-736. [PubMed: 112872]

4. Lamartiniere CA, Moore JB, Brown NM, Thompson R, Hardin MJ, Barnes S. Genistein suppresses mammary cancer in rats. Carcinogenesis. 1995; 16:2833-40. [PubMed: 7586206]

5. Lamartiniere CA, Murrill WB, Manzolillo PA, Zhang JX, Barnes S, Zhang X, Wei H, Brown NM. Genistein alters the ontogeny of mammary gland development and protects against mammary cancer in rats. Proc Soc Exper Biol Med. 1998; 217:358-64. [PubMed: 9492348]

6. Russo J, Russo IH. Toward a physiological approach to breast cancer prevention. Cancer Epidemiol Biomarkers Prev. 1994; 3:353-64. [PubMed: 8061586]

7. Russo IH, Russo J. Developmental stage of the rat mammary gland as a determinant of its susceptibility to 7,12-dimethylbenz[a] anthracene. J Natl Cancer Inst. 1978; 61:1439-1449. [PubMed: 102856] 
8. MacMahon B, Cole P, Lin TM, Lowe CR, Mirra AP, Ravnihar B, Salber EJ, Valaoras VG, Yuasa S. Age at first birth and breast cancer risk. Bull W H O. 1970; 43:209-221. [PubMed: 5312521]

9. Zhao S, Huang L, Wu J, Zhang Y, Pan D, Liu X. Vascular endothelial growth factor upregulates expression of annexin A2 in vitro and in a mouse model of ischemic retinopathy. Molecular Vision. 2009; 15:1231-1242. [PubMed: 19536308]

10. Sharma MC, Sharma M. The role of annexin II in angiogenesis and tumor progression: a potential therapeutic target. Curr Pharm Des. 2007; 13:3568-75. [PubMed: 18220793]

11. Syed SP, Martin AM, Haupt HM, Arenas-Elliot CP, Brooks JJ. Angiostatin receptor annexin II in vascular tumors including angiosarcoma. Hum Pathol. 2007; 38:508-13. [PubMed: 17239928]

12. Sharma MR, Koltowski L, Ownbey RT, Tuszynski GP, Sharma MC. Angiogenesis-associated protein annexin II in breast cancer: selective expression in invasive breast cancer and contribution to tumor invasion and progression. Exp Mol Pathol. 2006; 81:146-56. [PubMed: 16643892]

13. Zhang F, Zhang L, Zhang B, Wei X, Yang Y, Qi RZ, Ying G, Zhang N, Niu R. Anxa2 plays a critical role in enhanced invasiveness of the multidrug resistant human breast cancer cells. Journal of Proteome Research. 2009; 8:5041-5047. [PubMed: 19764771]

14. Erikson E, Shealy DJ, Erikson RL. Evidence that viral transforming gene products and epidermal growth factor stimulate phosphorylation of the same cellular protein with similar specificity. J Biol Chem. 1981; 256:11381-11384. [PubMed: 6271744]

15. Hunter T, Cooper JA. Epidermal growth factor induces rapid tyrosine phosphorylation of protein in A431 human tumor cells. Cell. 1981; 24:741-752. [PubMed: 6166387]

16. Olivier E, Soury E, Ruminy P, Husson A, Parmentier F, Daveau M, Salier JP. Fetuin-B, a second member of the fetuin family in mammals. Biochem J. 2000; 350:589-597. [PubMed: 10947975]

17. Brown WM, Saunders NR, Mollgard K, Dziegielewska KM. Fetuin, an old friend revisited. BioEssays. 1992; 14:749-755. [PubMed: 1285422]

18. Guo YL, Wang S, Colman RW. Kininostatin, an angiogenic inhibitor, inhibits proliferation and induces apoptosis of human endothelial cells. Arterioscler Thromb Vasc Biol. 2001; 21:14271433. [PubMed: 11557667]

19. Juarez JC, Guan X, Shipulina NV, Plunkett ML, Parry GC, Shaw DE, et al. Histidine-proline-rich glycoprotein has potent antiangiogenic activity mediated through the histidineproline- rich domain. Cancer Res. 2002; 62:5344-5350. [PubMed: 12235005]

20. Srinivas PR, Wagner AS, Reddy LV, Deutsch DD, Leon MA, Goustin AS, Grunberger G. Serum alpha 2-HS-glycoprotein is an inhibitor of the human insulin receptor at the tyrosine kinase level. Mol Endocrinol. 1993; 7:1445-1455. [PubMed: 7906861]

21. Hsu SJ, Nagase H, Balmain A. Identification of fetuin-B as a member of a cystatin-like gene family on mouse chromosome 16 with tumor suppressor activity. Genome. 2004; 47:931-946. [PubMed: 15499407]

22. Lay AJ, Jiang XM, Kisker O, Flynn E, Underwood A, Condron R, Hogg PJ. Phosphoglycerate kinase acts in tumor angiogenesis as a disulphide reductase. Nature. 2000; 408:869-873. [PubMed: 11130727]

23. Popanda O, Fox G, Thielmann HW. Modulation of DNA polymerase $\alpha, \delta$ and $\varepsilon$ by lactate dehydrogenase and 3-phosphoglycerate kinase. Biochim Biophys Acta. 1998; 1397:102-117. [PubMed: 9545551]

24. Duan Z, Lamendola DE, Yusuf RZ, Penson RT, Preffer FI, Seiden MV. Overexpression of human phosphoglycerate kinase 1 (PGK1) induces a multidrug resistance phenotype. Anticancer Res. 2002; 22:1933-41. [PubMed: 12174867]

25. Zhang DH, Tai LK, Wong LL, Chiu LL, Sethi SK, Koay ESC. Proteomic study reveals that proteins involved in metabolic and detoxification pathways are highly expressed in HER-2/neupositive breast cancer. Molecular \& Cellular Proteomics. 2005; 4:1686-1696. [PubMed: 16048908]

26. Silacci P, Mazzolai L, Gauci C, Stergiopulos N, Yin HL, Hayoz D. Gelsolin superfamily proteins: key regulators of cellular functions. Cell Mol Life Sci. 2004; 61:2614-2623. [PubMed: 15526166]

27. Asch HL, Head K, Dong Y, Natoli F, Winston JS, Connolly JL, Asch BB. Widespread loss of gelsolin in breast cancers of humans, mice and rats. Cancer Res. 1996; 56:4841-4845. [PubMed: 8895730] 
28. Lee HK, Driscoll D, Asch H, Asch B, Zhang PJ. Downregulated gelsolin expression in hyperplastic and neoplastic lesions of the prostate. Prostate. 1999; 40:14-19. [PubMed: 10344719]

29. Tanaka H, Shirkoohi R, Nakagawa K, Qiao H, Fujita H, Okada F, Hamada JI, Kuzumaki S, Takimoto M, Kuzumaki N. siRNA gelsolin knockdown induces epithelial- mesenchymal transition with a cadherin switch in human mammary epithelial cells. Int J Cancer. 2006; 118:1680-1691. [PubMed: 16217750]

30. Thor AD, Edgerton SM, Liu S, Moore DH, Kwiatkowski DJ. Gelsolin as a negative prognostic factor and effector of motility in erbB-2-positive epidermal growth factor receptor-positive breast cancers. Clin Cancer Res. 2001; 7:2415-2424. [PubMed: 11489821]

31. Yang J, Ramnath N, Moysich KB, Asch HL, Swede H, Alrawi SJ, Huberman J, Geradts J, Brooks JS, Tan D. Prognostic significance of MCM2, Ki-67 and gelsolin in nonsmall cell lung cancer. BMC Cancer. 2006; 6:203-210. [PubMed: 16882345]

32. Celli CM, Jaiswal AK. Role of GRP58 in mitomycin c-induced DNA cross-linking. Cancer research. 2003; 63:6016-6025. [PubMed: 14522930]

33. Flynn KM, Ferguson SA, Delclos KB, Newbold RR. Effects of genistein exposure on sexually dimorphic behaviors in rats. Tox Sci. 2000; 55:311-319.

34. Rowell C, Carpenter DM, Lamartiniere CA. Chemoprevention of breast cancer, proteomic discovery of genistein action in the rat mammary gland. J Nutr. 2005; 135:2953S-2959S. [PubMed: 16317154] 


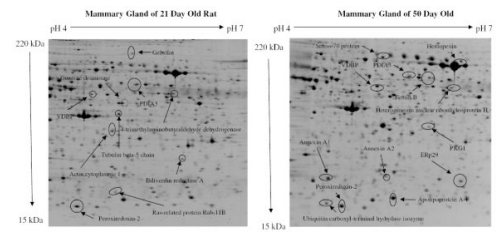

Figure 1.

Representative 2DE protein profiles with the protein spots marked as differentially regulated on mammary glands of 21 and 50 day old rats exposed to genistein. The identities of these spots identified by either MALDI-TOF-TOF or LC-ESI-MS/MS are presented in table 1 and 2. Total protein lysates were separated by isoelectric focusing using IPG DryStrip (180 mm, $\mathrm{pH} 4-7)$ and SDS-PAGE (12.5\%). SYPRO Ruby-stained gels were scanned using the Typhoon Imaging System (GE Healthcare) and analyzed using the SameSpots software (Nonlinear Dynamics, Newcastle, UK). 
A. Biological Processes

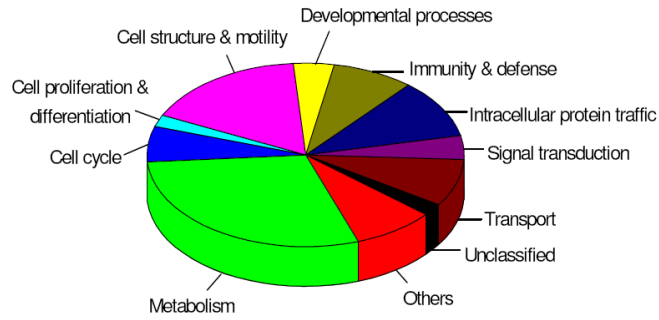

B. Signaling Pathway

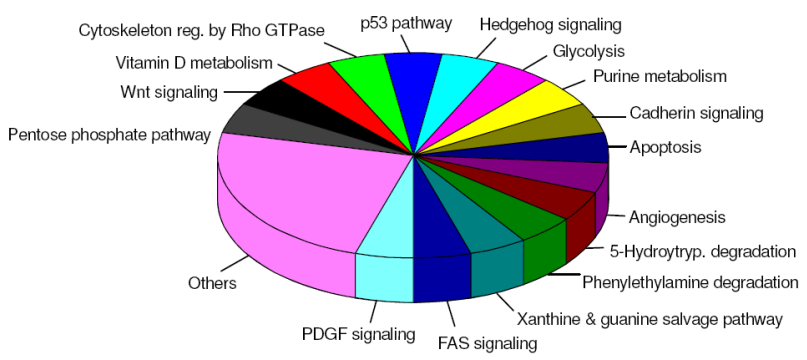

C. Cellular Localization

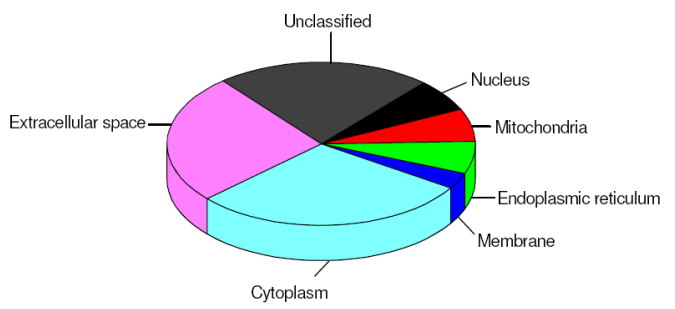

Figure 2.

Classification of proteins differentially regulated by prepubertal genistein exposure according to A) biological processes, B) signaling pathway and C) subcellular localization. The subset of 23 identified proteins were classified to their gene ontology groupings using the PANTHER classification system (www.pantherdb.org/). 


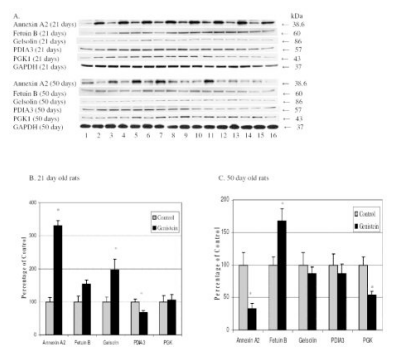

Figure 3.

Protein expressions of annexin A2, fetuin B, gelsolin, PDIA3 and PGK1 from mammary gland extracts of 21 and 50 day old rats exposed prepubertally to \pm genistein in the diet. A. Top panel depicts the western blots of mammary gland proteins from control and genistein-treated groups with different antibodies shown on left. Lanes 1, 3, 5, 7, 9, 11, 13 and 15, animals on control diet; Lanes 2, 4, 6, 8, 10, 12, 14 and 16, animals on genistein containing diet from birth until day 21 postpartum.

B. Densitometric values from 21 day old rats were reported as a percentage of the controls \pm SEM. $* p<0.05$, compared with controls.

C. Densitometric values from 50 day old rats were reported as a percentage of the controls \pm SEM. $* p<0.05$, compared with controls. 
A.

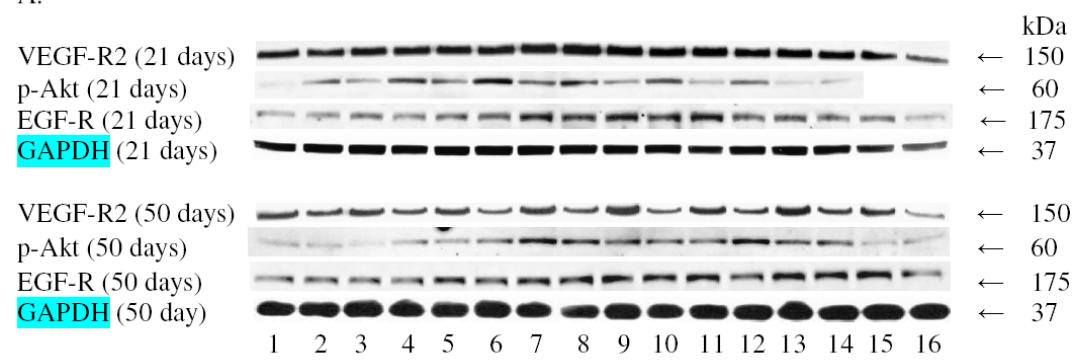

B. 21 day old rats

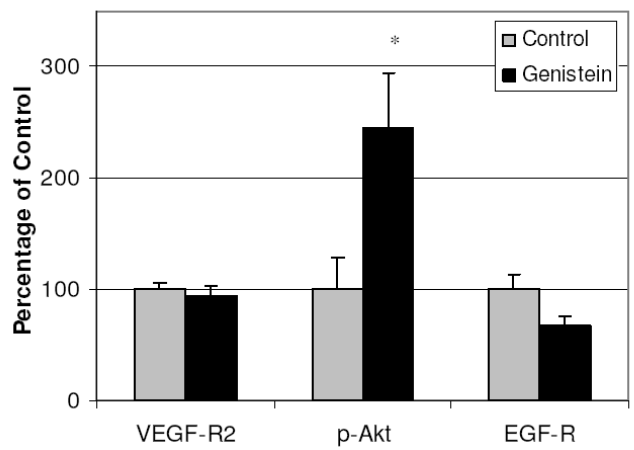

C. 50 day old rats

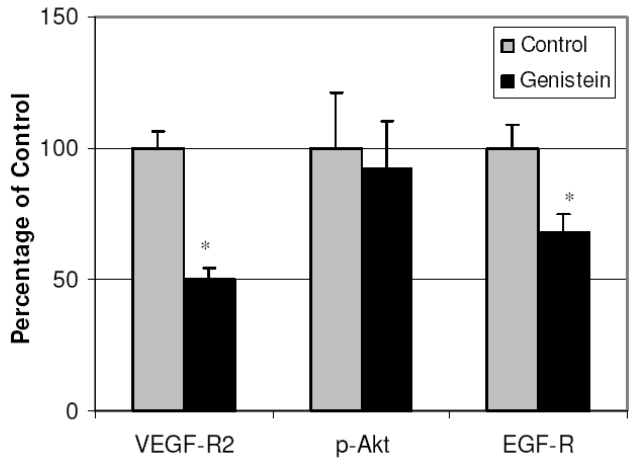

Figure 4.

Protein expressions of VEGF-R2, p-Akt and EGF-R from mammary gland extracts of 21 and 50 day old rats exposed prepubertally to \pm genistein in the diet.

A. Top panel depicts the western blots of mammary gland protein from control and genistein-treated groups with different antibodies shown on left. Lanes 1, 3, 5, 7, 9, 11, 13 and 15, animals on control diet; Lanes 2, 4, 6, 8, 10, 12, 14 and 16, animals on genistein containing diet from birth until day 21 postpartum.

B. Densitometric values from 21 day old rats were reported as a percentage of the controls \pm SEM. $* p<0.05$, compared with controls.

C. Densitometric values from 50 day old rats were reported as a percentage of the controls \pm SEM. $* p<0.05$, compared with controls. 


\section{Control}

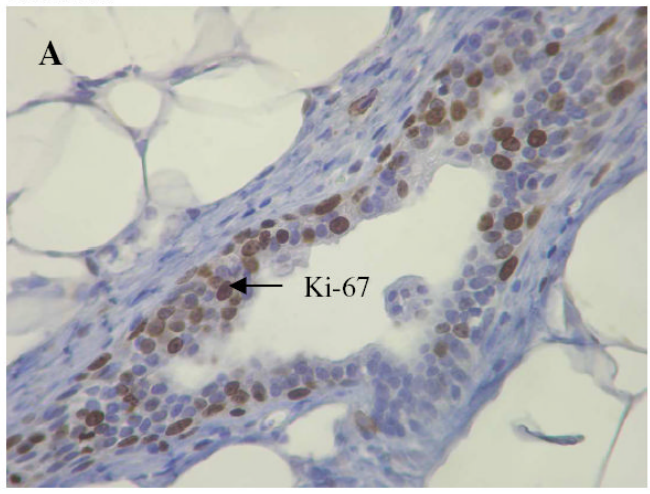

Genistein treated

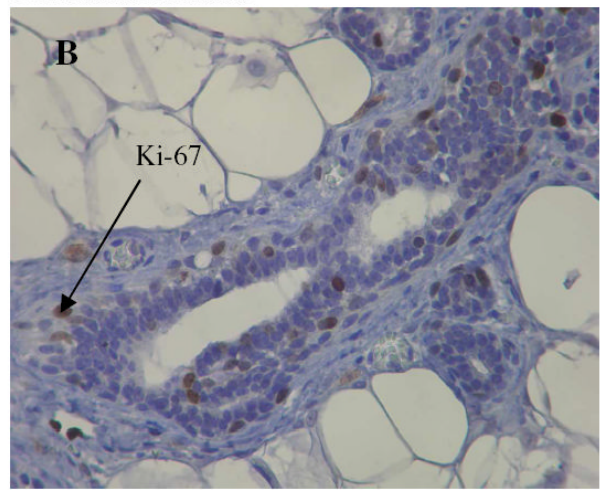

C

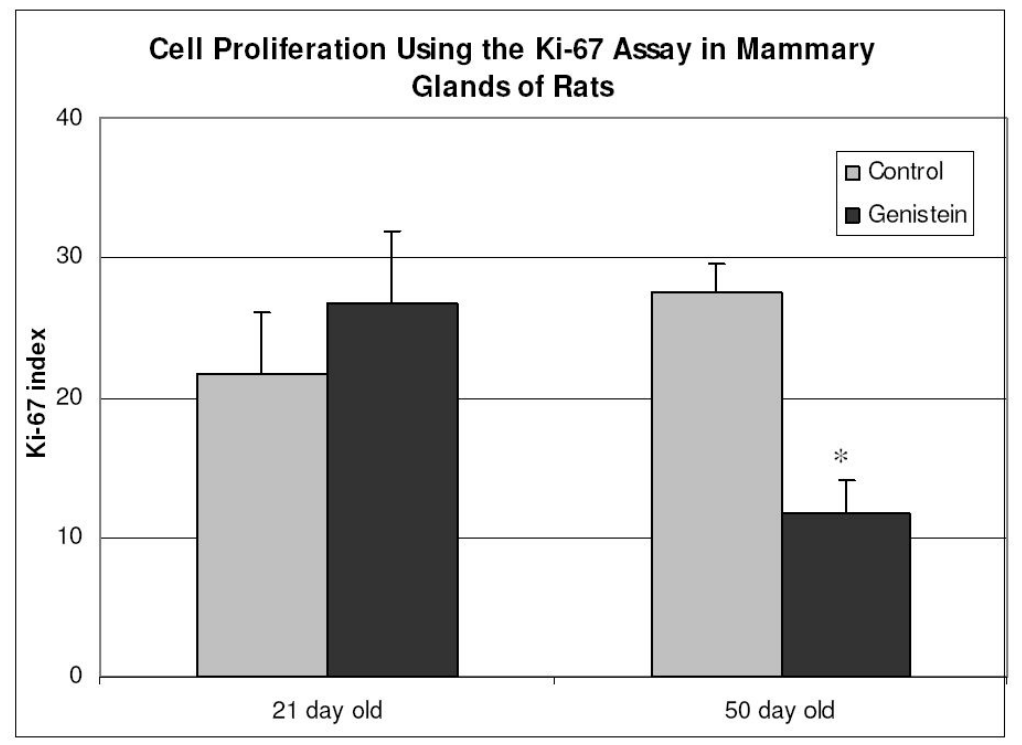

Fig. 5.

Ki-67 expression in the mammary glands of 21-day and 50 day-old rats whose dams were fed AIN-76A diet (control) or AIN-76A diet supplemented with $250 \mathrm{mg}$ genistein per $\mathrm{kg}$ diet from day 1 until day 21 postpartum. Sections (A and B) represent immunohistochemical staining of Ki-67 in mammary glands of 50 day old rats treated with control (A) and genistein (B), respectively (x 40). Arrows point to positively stained cells. (C) Cell proliferation index in mammary glands of 21 day and 50 day old rats. Six mammary gland samples $(n=6)$ were analyzed per treatment group. $* \mathrm{P}<0.001$ compared with control group. 

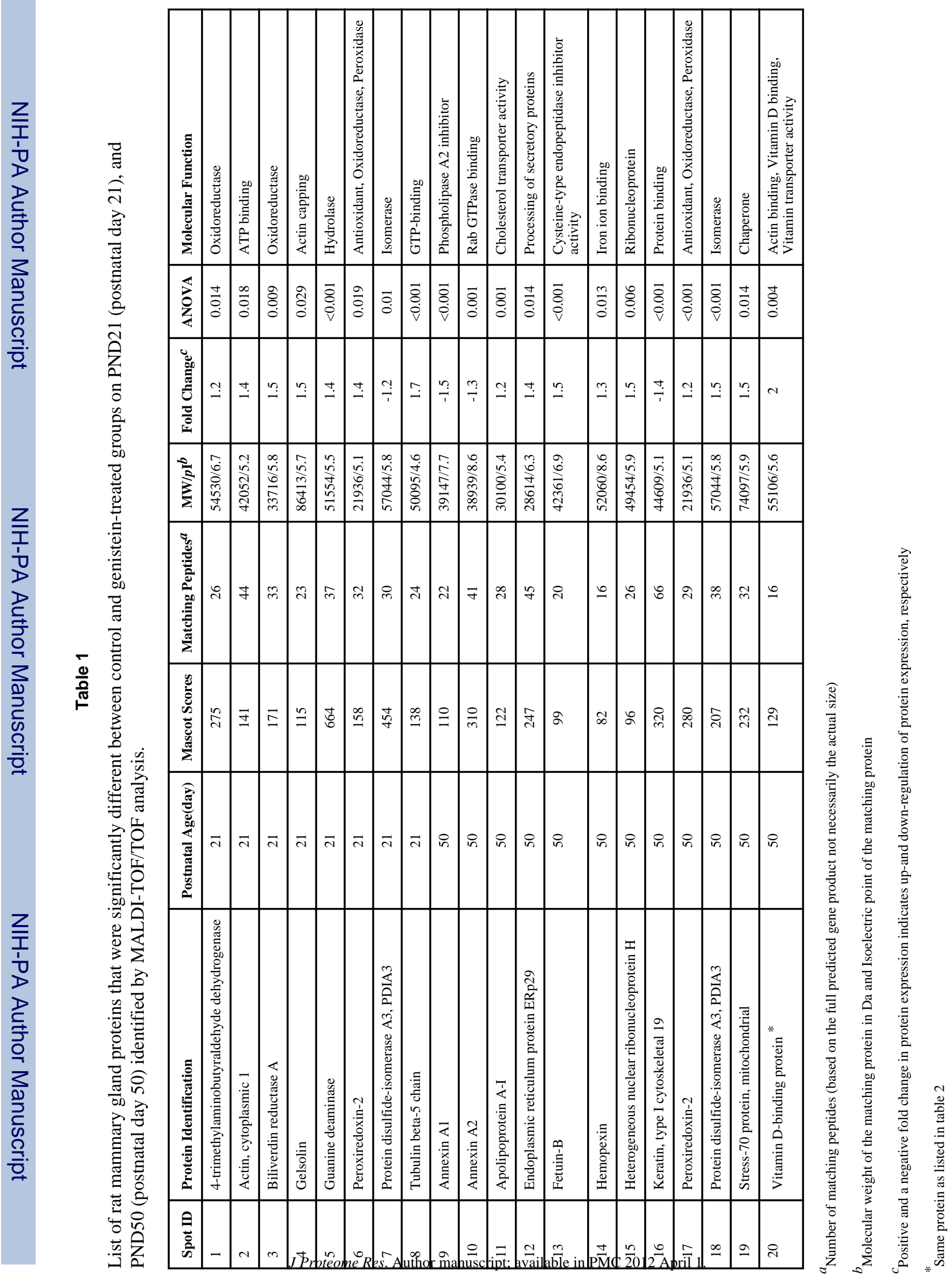


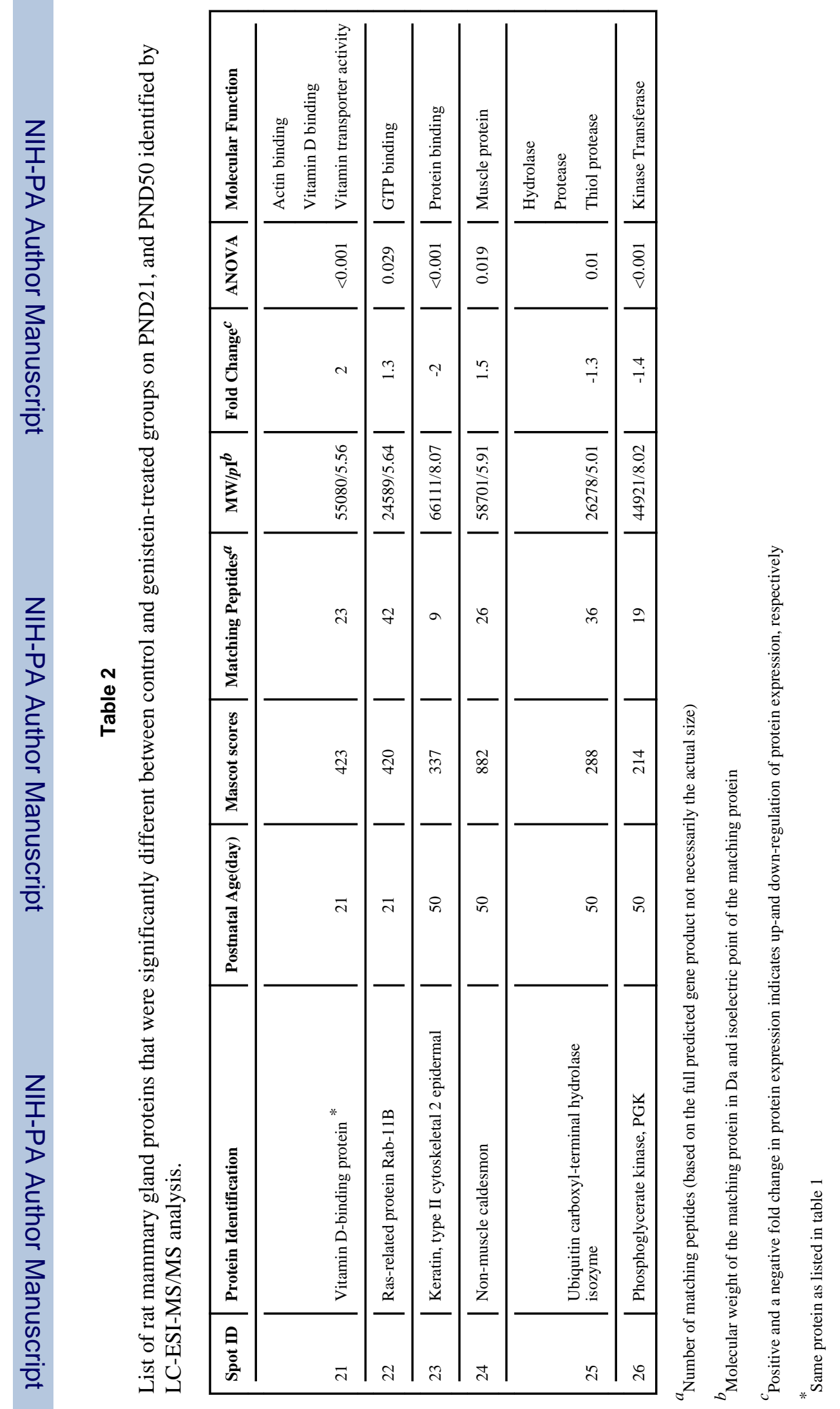

J Proteome Res. Author manuscript; available in PMC 2012 April 1. 